\title{
Primary Gastrointestinal Follicular Lymphomas: A Prospective Study of 31 Patients with Long-term Follow-up Registered in the French Gastrointestinal Lymphoma Study Group (GELD) of the French Federation of Digestive Oncology (FFCD)
}

\author{
Tamara Matysiak-Budnik ${ }^{1,2}$, Philippe Jamet ${ }^{1}$, Nicolas Chapelle ${ }^{1,3}$, Bettina Fabiani ${ }^{4}$, Paul Coppo ${ }^{5}$, and Agnès \\ Ruskoné-Fourmestraux ${ }^{6,7}$ \\ ${ }^{1}$ IMAD, Department of Gastroenterology and Digestive Oncology, Hotel Dieu Hospital, ${ }^{2}$ UMR Inserm 1235 and ${ }^{3}$ INSERM UMR 1064, \\ University of Nantes, Nantes, Departments of ${ }^{4}$ Pathology, ${ }^{5}$ Hematology, and ${ }^{6}$ Gastroenterology, Saint Antoine Hospital AP-HP, Paris, \\ and ${ }^{7}$ French Federation of Digestive Oncology, Dijon, France
}

\section{Article Info}

Received July 1, 2021

Revised October 22, 2021

Accepted October 29, 2021

Published online March 7, 2022

Corresponding Author

Tamara Matysiak-Budnik

ORCID https://orcid.org/0000-0003-0780-6261

E-mail tamara.matysiakbudnik@chu-nantes.fr
Background/Aims: Primary gastrointestinal follicular lymphomas (PGFL) are very rare. Our aim was to analyze the clinical features, management, and long-term outcomes in a prospective series of patients diagnosed with PGFL.

Methods: All adult patients with PGFL, consecutively enrolled into the multicenter French study between 1990 and 2017, were evaluated and followed up prospectively after undergoing a complete work-up. Clinical, pathological and endoscopic features, as well as treatment outcomes, were analyzed.

Results: Thirty-one patients (16 men, median age 62 years, range 33 to 79 years) with PGFL were included. The median follow-up was 92 months (range, 6 to 218 months). In the majority of patients $(n=14)$, lymphoma was incidentally diagnosed during endoscopy. Otherwise, the most frequent circumstances of diagnosis were abdominal pain $(n=7)$ and dyspepsia $(n=5)$. The duodenum was the most common site of involvement $(n=19)$ and multifocal localizations were seen in seven patients (22\%). The most frequent first line strategy was surveillance applied in 22 patients $(71 \%)$, of whom nine reached spontaneous, complete remission and 11 had stable disease. Seven patients $(23 \%)$ received chemotherapy as first line treatment, and two underwent resection. Of seven patients who received chemotherapy, four achieved complete remission. In three patients, transformation into a high-grade lymphoma occurred.

Conclusions: The diagnosis of PGFL is frequently fortuitous. The most common localization is in the duodenum. The disease has an indolent course and a good prognosis, however, rare cases of transformation into aggressive high-grade lymphoma may occur. An appropriate characterization and follow-up of these lymphomas is mandatory for their optimal management. (Gut Liver 2022;16:207-215)

Key Words: Primary gastrointestinal follicular lymphoma; Follicular lymphoma; Transformation; Clinical management; Follow-up

\section{INTRODUCTION}

The gastrointestinal tract is the most common site of extranodal non-Hodgkin's lymphomas. Primary gastrointestinal lymphomas comprise several different entities, which have been better characterized in the last decades due to the development of immunohistochemical and molecular diagnostic methods. Primary gastrointestinal follicular lymphomas (PGFL) represent a very rare entity, whose frequency in different clinicopathological series of primary gastrointestinal lymphomas varied from 3\% to $13 \% .^{1-4}$ The clinical and biological characteristics of these 
lymphomas are not well known, and have been described mainly through clinical cases ${ }^{5-9}$ and some clinicopathological series, usually retrospective, reported in the literature..$^{10-18}$ Due to their rarity and lack of randomized trials, the treatment of PGFL is not standardized. Data obtained from much more frequent nodal follicular lymphomas (FL) have been extrapolated to PGFL. However, it is now becoming clear that these two entities, primary nodal and primary digestive FL, differ considerably in terms of their physiopathology and prognosis. ${ }^{16,19}$ Our aim was to describe the clinical characteristics and treatment outcome of the patients diagnosed with PGFL included in a large, prospective French study, carried out by the French Gastrointestinal Lymphoma Study Group (GELD; Groupe d'Etude des Lymphomes Digestifs), with a long-term follow-up allowing for the analysis of the long-term outcome of this rare disease.

\section{MATERIALS AND METHODS}

\section{Patients}

From January 1990 to June 2017, all cases of PGFL were consecutively enrolled into a prospective, multicenter study on gastrointestinal lymphomas conducted by the French GELD under the auspices of the French Federation of Digestive Oncology (FFCD; Fédération Francophone de Cancérologie Digestive). Gastroenterologists from eight hospital centers (Villemomble Hospital Center, Longjumeau Hospital Center, St Germain en Laye Hospital Center, University Hospital of Anglet, Bussy St Georges Hospital Center, University Hospital of Blanc Mesnil, University Hospital of Reims, Saint Antoine University Hospital AP-HP), recruited the patients for this study. All data were collected by the local investigators according to the study chart, and transferred to the coordination Center at Saint Antoine Hospital in Paris, where they were centralized and analyzed.

The primary intestinal character of the lymphoma was defined according to Lewin et al. ${ }^{20}$ as either predominant intestinal lesion without previous peripheral lymphadenopathy or any lymphoma whose clinical presentation was related to intestinal involvement. Patients with nongastrointestinal primary FL were excluded from this study. The diagnosis of PGFL was based on the World Health Organization (WHO) classification. ${ }^{21}$ This classification distinguishes different types of $\mathrm{FL}$, and in particular recognizes primary gastrointestinal tract FL as a variant named duodenal-type FL. It also underlines its clinical and biological features with an excellent outcome, even without treatment.
Informed written consent was obtained from all patients and the study was approved by the CCPPRB Hospital Hotel Dieu AP-HP (approval number: 98421).

According to the study chart, all data on the patients' medical history, symptoms and circumstances of diagnosis, had to be recorded. Incidental lymphoma was defined as diagnosis made during screening endoscopy or endoscopy performed for symptoms unlikely to be related to lymphoma (mainly gastroesophageal reflux).

\section{Histology and immunohistochemistry}

The histology of each tumor, based on endoscopic biopsies and/or surgical material, was reviewed by at least two expert pathologists. Tumors were classified according to the WHO classification of tumors of hematopoietic and lymphoid tissues. ${ }^{21}$ Immunohistochemical analysis was performed on paraffin-embedded biopsies and included staining with anti-CD20, anti-CD3 antibodies to confirm the B-cell nature of the lymphoid infiltrate. Additional staining with anti-CD5, anti-CD10, anti-cyclin D1, antiBCL-2, and MIB-1 (Ki-67) was used to further characterize and classify the lymphoma.

The histological diagnosis of FL was confirmed on the basis of the presence of neoplastic follicles in the mucosa/ submucosa, made up of centrocytes and very rare centroblasts, of a typical for FL phenotype (CD20+, BCL2+, CD10+, CD5-, CD43- and cyclin D1-), and low proliferative index (Ki-67). Neoplastic B cells also infiltrate the lamina propria outside the follicles, and this is best illustrated with CD20 immunostaining.

\section{Staging}

A complete work-up was performed in all patients, including physical examination with WHO performance status assessment, hematological and biochemical tests (liver function test, full blood count, serum protein immunofixation, lactate dehydrogenase [LDH] levels, $\beta 2$ microglobulin levels, human immunodeficiency virus, and hepatitis B and C viral serologies). Thoracic-abdominal-pelvic computed tomography, as well as upper and lower endoscopy with multiple biopsies of the tumor and multilevel systematic biopsies, were performed in all patients. Bone marrow biopsy was performed in all but six patients, that were more recently included, in whom peripheral blood lymphocyte phenotyping was performed instead of a bone marrow biopsy. Since 2014, an integrated positron emission tomography with 2-deoxy-2- $\left[{ }^{18} \mathrm{~F}\right]$ fluoro-D-glucose/ positron emission tomography-computed tomography $\left({ }^{18} \mathrm{~F}\right.$-FDG-PET/CT) was performed.

Extension of the disease was defined according to the Ann Arbor staging system, modified by Musshoff. ${ }^{22}$ Lym- 
phoma was classified as multifocal when more than one digestive segment was affected. In case of multiple lesions (nodular and/or polypoid) situated within one digestive segment, the lymphoma was considered as unifocal stage IE. Stage IV was declared in case of bone marrow involvement, or in the presence of extra-digestive localization (including clonal B-cell population in peripheral blood).

\section{Treatment and response to treatment}

All the administered treatments and treatment outcomes had to be recorded in a prospective manner by the investigator at each center. Therapeutic strategy for each patient was left at the discretion of the investigator medical team (by taking into account the age of the patient, clinical presentation, and validated tumor burden criteria ${ }^{12,23}$ ) and had to be registered. The follow-up data also had to be prospectively collected.

Follow-up (in case of surveillance) or response to treatment (at the end of the initial treatment) were assessed by a work-up identical to the pre-therapeutic work-up for involved sites. Complete remission was defined as complete (macroscopic and microscopic) disappearance of the lymphoma in all sites initially involved and the absence of new tumors, confirmed by serial negative endoscopic biopsies from all involved sites. Further evaluations were planned every year for at least 10 years. Other treatment outcomes

Table 1. Demographic and Clinical Characteristics of All the Patients with Primary Gastrointestinal Follicular Lymphoma

\begin{tabular}{lc}
\hline \multicolumn{1}{c}{ Characteristics } & Value \\
\hline No. of patients & 31 \\
Age, median (range), yr & $62(33-79)$ \\
Sex, male/female & $16 / 15$ \\
Circumstances of diagnosis & \\
Fortuitous diagnosis & 14 \\
Abdominal/epigastric pain* & 7 \\
Dyspepsia & 5 \\
Rectal bleeding & 4 \\
Alteration of general status & 1 \\
Endoscopic features & \\
Macro-/micronodular & 17 \\
Polyposis & 6 \\
Tumor ${ }^{\dagger}$ & 5 \\
Polyp & 2 \\
Ulceration & 1 \\
Anatomical distribution of lesions & \\
Unifocal & 24 \\
Multifocal & 7 \\
Stage & \\
IE & 23 \\
IIE & 3 \\
IV & 5
\end{tabular}

*Including 1 with intestinal obstruction; ${ }^{\dagger}$ Including 2 with stenosis; ${ }^{\ddagger}$ According to Ann Arbor staging system modified by Musshoff. ${ }^{22}$ were classified, according to the defined criteria, as partial remission, stability of the disease, progression of the disease, and death.

\section{Statistical analysis}

Statistical analyses were performed using SAS 9.3 (SAS Institute Inc., Cary, NC, USA). The survival rates were calculated using the Kaplan-Meier method. Overall survival was calculated from the date of diagnosis to the date of the final follow-up or death from any cause. Progression-free survival was calculated from the date of diagnosis to the date of disease progression, relapse or the date of the final follow-up.

\section{RESULTS}

\section{Patient characteristics}

From June 1990 to January 2017, 31 cases of PGFL entered the national study of GELD. There were 16 men and 15 women, median age at diagnosis was 62 years (range, 33 to 79 years).

Demographic and clinical features of the patients are shown in Table 1. The most frequent circumstances of diagnosis were fortuitous diagnosis during endoscopy performed for other reasons $(n=14)$, followed by abdominal pain $(n=7)$, dyspepsia $(n=5)$, and rectal bleeding $(n=4)$. All patients were in good general status at diagnosis, with a WHO score of $0-1$. The most frequent initial lymphoma location was the duodenum (19 cases, including two with concomitant ileum localizations), and multiple localizations were found in seven patients (Tables 1,2).

The most frequent endoscopic features were micronodular or polypoid appearance (23 patients) (Fig. 1).

At diagnosis, patients had stage IE (23 cases), stage IIE (three cases) or stage IV (five cases) disease. Bone marrow biopsy was performed in 25 out of 31 patients, and was positive in three patients with stage IV disease. In the remaining two patients with stage IV disease and negative bone marrow biopsy, a clonal B lymphocyte population was found in peripheral blood. An ${ }^{18} \mathrm{~F}$-FDG-PET/CT was

Table 2. Lymphoma Site Distribution in Patients with Primary Gastrointestinal Follicular Lymphoma

\begin{tabular}{lcc}
\hline \multicolumn{1}{c}{ Variable } & Unifocal $(\mathrm{n}=24)$ & Multifocal $(n=7)$ \\
\hline Stomach & 1 & \\
Duodenum (D1, D2, D3) & 17 & \\
Jejunum & 1 & $2^{*}$ \\
lleum & 3 & \\
Colon/rectum & 2 & $5^{\dagger}$ \\
\hline
\end{tabular}

*With ileum involvement; ${ }^{\dagger}$ With gastric and small bowel involvement. 
performed in the five most recently included patients and in all cases confirmed a localized disease. Serum LDH and $\beta 2$-microglobulin were evaluated in all patients: $\beta 2$ microglobulin levels were increased in one patient with stage IV lymphoma, while LDH levels were normal in all patients.

\section{Histopathology}

Histopathological diagnosis was made in all but two cases based on the analysis of biopsies obtained during en-
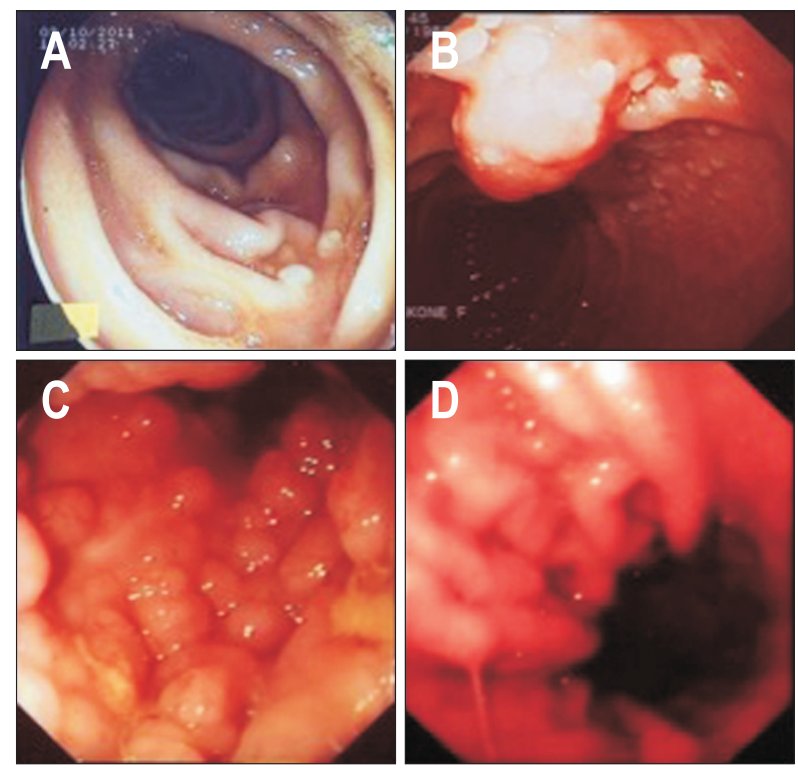

Fig. 1. Endoscopic findings in patients with primary gastrointestinal follicular lymphomas. Duodenal follicular lymphoma: micronodular appearance of distal duodenal mucosa (A, B). Rectal follicular lymphoma: polyposis-like appearance of rectal mucosa (C). Gastric follicular lymphoma: enlarged pseudopolypoid gastric folds (D). doscopy. In the two remaining cases, diagnosis was made on surgical or endoscopic resection specimens. The lymphoid proliferation consisted of round lymphoid follicles, located in the mucosa and submucosa, composed almost uniformly of centrocytes with infrequent centroblasts. The neoplastic follicles were CD20+, CD10+, BCL6+ and had a low Ki-67 proliferative index (Fig. 2).

\section{Treatment and outcome}

As initial management, 22 patients, all with stage IE FL, were offered a "watch and wait" strategy (Table 3). Among these 22 patients, 16 had FL restricted to the duodenum, one had duodenal FL with a second localization in the ileum, one had gastric FL, one ileal FL, one jejunal FL, one colonic FL, and one had rectal FL with a second gastric localization. Nine patients (including seven with duodenal, one with colonic, and one with ileal lymphoma) achieved a spontaneous, complete remission after a median time of 5 years (range, 3 to 13 years), 11 had stable disease over time, and one, with rectal and gastric localizations, showed a progression after 1 year requiring chemotherapy. One patient was lost to follow-up.

Seven patients (six with multifocal disease and one with a localized duodenal bulky disease) received upfront chemotherapy. Detailed information about the regimen was available for six patients: R-CHOP (rituximab-cyclophosphamide-doxorubicin-vincristine-prednisone) in two cases and R-CVP (rituximab-cyclophosphamide-vincristineprednisone), CHVP-IFN (cyclophosphamide-doxorubicinvindesine-prednisone-interferon), cyclophosphamide, AVmCP (doxorubicin-teniposide-cyclophosphamideprednisone) in one of the remaining cases each. Out of these seven patients, four achieved a complete remission.
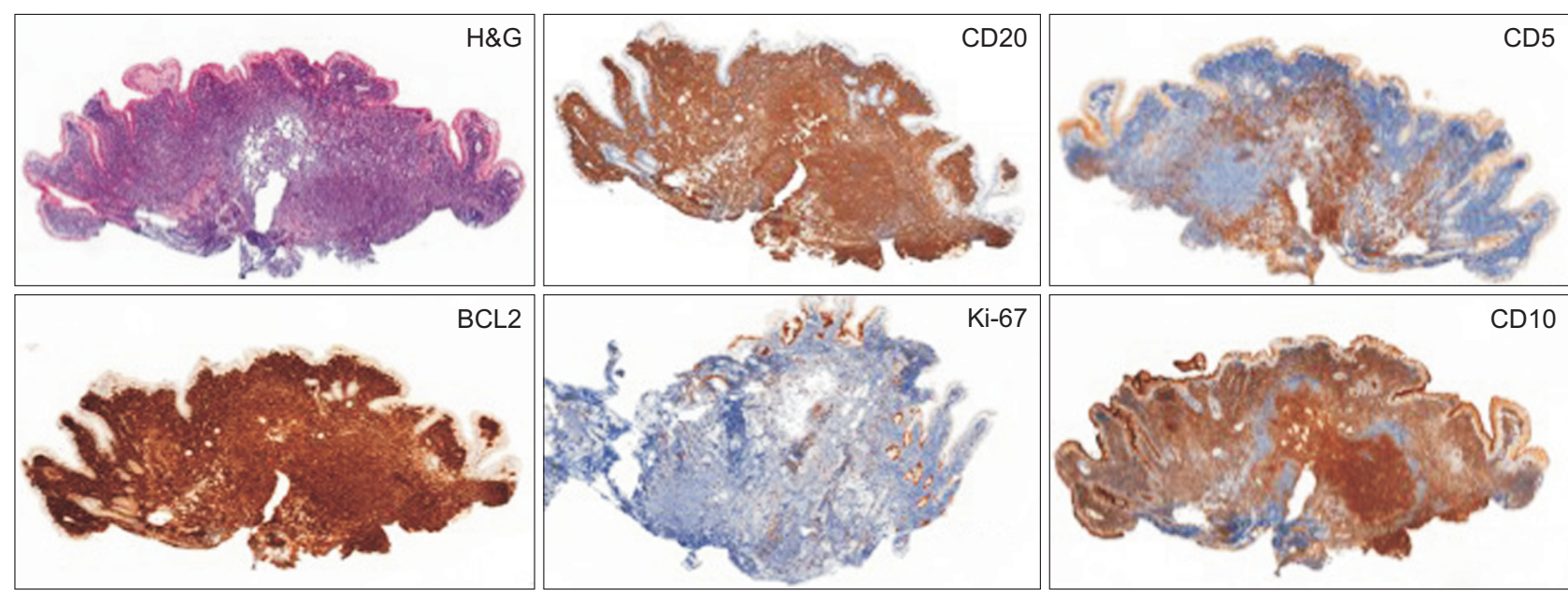

Fig. 2. Histology and immunohistochemistry staining images in patient with a primary duodenal follicular lymphoma. Neoplastic follicles of the duodenal mucosa (H\&E, ×50). Neoplastic cells are also present in the lamina propria, outside the follicles. Tumor cells are CD20+, CD10+, BCL2+, and CD5- (small tumor CD5+ lymphocytes around the nodules), and have a very low Ki-67 proliferation index (immunohistochemistry, $\times 50$ ). 
Matysiak-Budnik T, et al: Primary Gastrointestinal Follicular Lymphomas

Table 3. Initial Treatment According to the Stage of the Disease in Patients with Primary Gastrointestinal Follicular Lymphomas

\begin{tabular}{ccccc}
\hline Stage* $^{*}$ & Surveillance & Chemotherapy & Surgery & Endoscopic resection \\
\hline IE & 22 & 0 & 0 & 1 \\
IIE & 0 & 2 & 1 & 0 \\
IV & 0 & 5 & 0 & 0 \\
\hline
\end{tabular}

*According to Ann Arbor staging system modified by Musshoff. ${ }^{22}$

Table 4. Final Outcome in 31 Patients with Primary Gastrointestinal Follicular Lymphomas

\begin{tabular}{lcccccc}
\hline \multicolumn{1}{c}{ Treatments } & No. of patients & CR & SD & PD & PR & Unknown \\
\hline Surveillance (no treatment) & 20 & 9 & 10 & 0 & 0 & 1 \\
Surveillance+chemotherapy & 2 & 1 & 0 & 0 & 1 & 0 \\
Chemotherapy only & 7 & 4 & 0 & $3^{*}$ & 0 & 0 \\
Surgery+chemotherapy & 1 & 1 & 0 & 0 & 0 & 0 \\
Endoscopic resection & 1 & 1 & 0 & 0 & 0 & 0 \\
\hline
\end{tabular}

Data are final outcome after a median follow-up of 92 months (range, 6-218 months).

$\mathrm{CR}$, complete remission; $\mathrm{SD}$, stable disease; $\mathrm{PD}$, progressive disease; $\mathrm{PR}$, partial regression.

*Transformations into high-grade lymphoma.

Table 5. Final Outcome after All Lines of Treatment or Surveillance ("Watch and Wait” Strategy) in Patients with Primary Gastrointestinal Follicular Lymphomas

\begin{tabular}{cccccc}
\hline Stage* & No. of patients & CR & SD & PD & Unknown \\
\hline IE & 23 & 11 & 10 & 1 & 1 \\
IIE & 3 & 1 & 1 & 1 & 0 \\
IV & 5 & 3 & 0 & 2 & 0 \\
\hline
\end{tabular}

Data are final outcome after a median follow-up of 92 months (range, 6-218 months), according to the initial stage of the disease.

$\mathrm{CR}$, complete remission; $\mathrm{SD}$, stable disease; $\mathrm{PD}$, progressive disease.

*According to Ann Arbor staging system modified by Musshoff. ${ }^{22}$

From these four patients, two relapsed after 5 and 9 years after remission, respectively, but achieved a second durable remission after a salvage treatment. The three other patients, two of whom achieved a partial remission, experienced a transformation into a high-grade lymphoma.

For the two remaining patients, one presented a localized but obstructive duodenal lymphoma requiring surgery. He subsequently received chemotherapy followed by autologous stem cell transplantation; he is still in complete remission 11 years after the treatment. The second patient had a complete endoscopic resection of a colonic polyp which corresponded to FL. He is still in complete remission after an 8-year follow-up.

After a median follow-up of 92 months (range, 6 to 218 months), the overall survival was good, with over $80 \%$ of patients still alive 10 years after diagnosis and $70 \%$ of them without disease progression (Tables 4, 5 and Fig. 3). Among the three patients who died during the follow-up, two experienced transformation into high-grade lymphoma and died from this lymphoma, and one patient developed a metastatic neuroendocrine carcinoma and died from this carcinoma.

\section{Transformation into high-grade lymphoma}

In three patients (9\%), a transformation into a highgrade lymphoma was observed, 1,4 , and 9 years after diagnosis, respectively. Two of these patients initially presented a multifocal FL, stage IE and IIE, respectively, and one stage IV lymphoma with a voluminous duodenal mass.

\section{Secondary cancers}

Three patients from our cohort developed another cancer during the follow-up period: one gastric adenocarcinoma, one metastatic neuroendocrine tumor of unknown origin, and one gastric mucosa associated lymphoid tissue lymphoma, 16, 9, and 10 years after diagnosis of FL, respectively.

\section{DISCUSSION}

We report here the largest, prospective series of patients with PGFL published so far, giving a detailed description of this rare disease over a long follow-up period. The number of patients included (31 during a 27-year period) certainly 
A

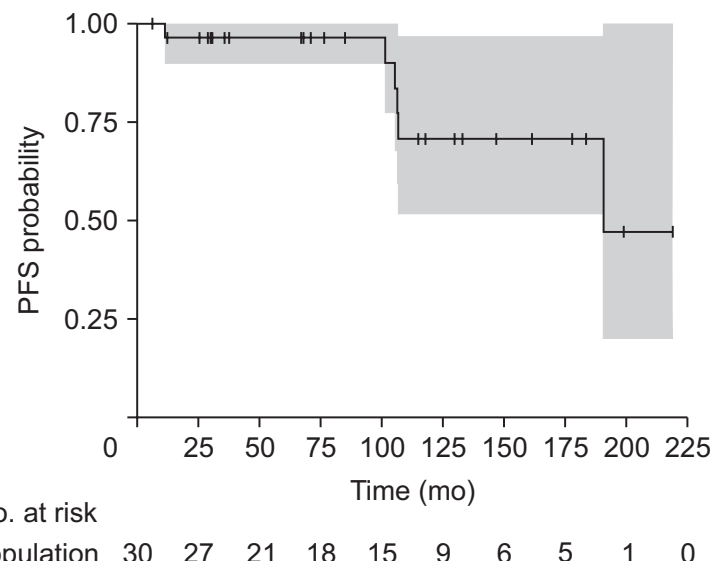

B

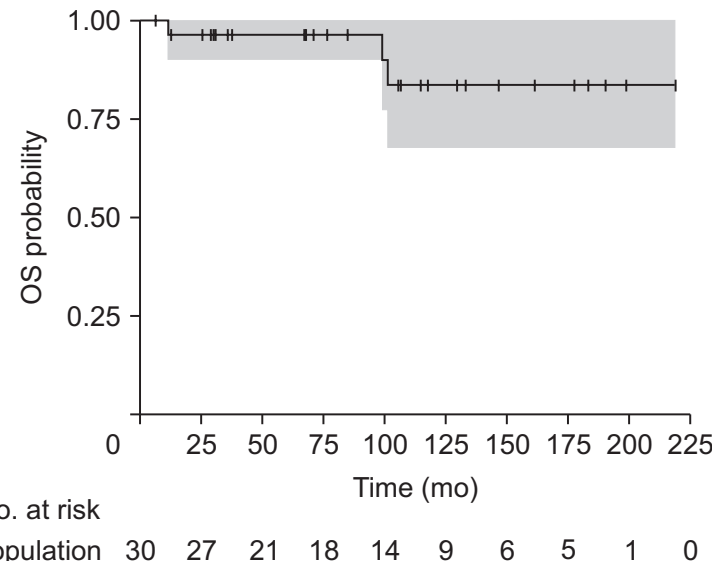

Fig. 3. PFS (A) and OS (B) in patients with primary gastrointestinal follicular lymphomas. The grey area correspond to the $95 \%$ confidence interval of estimated number of patients with PFS and OS.

PFS, progression-free survival; OS, overall survival.

does not reflect the real incidence/prevalence of PGFL in France which is probably higher.

Our first finding is that in the majority of cases, especially those with a single duodenal localization, the diagnosis of PGFL is made fortuitously, before nonspecific symptoms, apparently not related to the lymphoma. The usually limited character of the lesions explains the good WHO performance status (0-1) of these patients.

In nodal FL, the FL international prognostic index, based on five adverse prognostic factors (age above 60 years, Ann Arbor stage III or IV, increased LDH levels, hemoglobin levels inferior to $12 \mathrm{~g} / \mathrm{dL}$, and presence of more than 4 nodal sites of disease), has been validated as predictive of survival. ${ }^{24}$ However, this index appears to be less useful in patients with PGFL who are usually in a better clinical condition. ${ }^{16,19}$ Therefore, in the present study, we used the WHO index to assess the general status of the patients.

In terms of localization, we confirm previous reports that duodenum is a preferential site of PGFL, although this observation could be the result of better accessibility of the proximal intestine for exploration by endoscopy. Indeed, the relatively high frequency of duodenal FL diagnosis in clinical series coming from Japan, where upper endoscopy is performed very commonly, may be in favor of the second hypothesis. ${ }^{10,11,17}$

The multifocal character of the lesions $(23 \%$ in our series) is another typical feature of PGFL. ${ }^{13,14}$

Another interesting point is the low disease stage (IE or IIE) in the majority of the patients at diagnosis, while patients with nodal FL more typically have stage III or IV disease. ${ }^{16,19,25}$ In our series, among the five patients with stage IV lymphoma, only two had bone marrow involvement, while in the three remaining cases, stage IV resulted from the presence of distant nodes or organ involvement and/or the presence of clonal B lymphocytes in peripheral blood.

The ${ }^{18} \mathrm{~F}$-FDG-PET/CT has recently been shown to be a useful tool for staging of FL. ${ }^{26,27}$ Its value in gastrointestinal FL has not yet been determined. In our series, only the most recently included patients could benefit from ${ }^{18} \mathrm{~F}$ FDG-PET/CT that confirmed that the disease was localized in all cases. In PGFL, the ${ }^{18} \mathrm{~F}$-FDG-PET/CT could be of particular interest to eliminate small intestinal localizations since other techniques, like enteroscopy or videocapsule endoscopy, are not always available and their diagnostic performance in small intestinal FL remains to be confirmed.

The initial management in the majority of our patients (70\%), with stage IE FL, was a "watch and wait" strategy, as proposed by the general consensus for nodal FL. ${ }^{23,27-29}$ In patients with asymptomatic intestinal FL, it has been shown that rituximab-based chemotherapy does not improve prognosis compared to the "watch and wait" strategy. ${ }^{30}$

Radiotherapy is also a valuable option in PGFL. However, studies providing strong level of evidence for the treatment of PGFL are scarce; consequently, our attitude was largely practitioner-dependent. On the one hand, some series of patients with duodenal and intestinal FL treated with radiotherapy provided encouraging results; on the other hand, it is also recognized that irradiation of the small intestine may be toxic and therefore of limited use in some centers. ${ }^{31}$ Moreover, in patients with localized duodenal lesions and candidates for RT, a watch and wait attitude is also an acknowledged attitude, ${ }^{18}$ that we have preferred here. 
Important information coming from our study is that localized duodenal FL can spontaneously completely regress. ${ }^{15,32}$ Based on our results, we suggest that the best candidates for surveillance are patients with localized duodenal FL with low tumor burden.

Prognosis of PGFL is usually very good, as confirmed by our study showing that over $80 \%$ of patients were alive 10 years after diagnosis. However, cases of transformation into high-grade lymphoma may occur, which considerably worsens the prognosis of the disease. ${ }^{33}$ In our cohort, three patients (9\%) experienced transformation with poor prognosis, probably due to delayed diagnosis. These patients had different stages of the disease at diagnosis (IE, IIE, and IV, respectively), but they were all symptomatic and had a great tumor burden at diagnosis, with multifocal lesions in two of them and a large, duodenal tumor mass in the third patient. Unfortunately, we do not have information about the molecular profile of these tumors, which may be an interesting tool in the future to predict aggressive outcome of the disease. ${ }^{34}$ However, our data suggest, that independently of the disease stage at diagnosis, patients with symptoms and a large tumor mass should be given particular attention since they present a higher risk of transformation into a high-grade lymphoma.

The association between lymphoid malignancies and an increased risk of other malignancies is well recognized. ${ }^{35,36}$ Here, we report evidence that such association also exists in PGFL (9\% of patients in our series). Consequently, as PGFL patients may experience a very long follow-up, practitioners should pay attention to this possible complication.

In conclusion, PGFL are frequently localized in the duodenum and have an indolent course, and may regress completely without any treatment. However, transformation into high-grade lymphomas or secondary malignancies may occur. Risk factors for such complications need to be identified in these patients who are generally followed up for many years.

\section{CONFLICTS OF INTEREST}

No potential conflict of interest relevant to this article was reported.

\section{ACKNOWLEDGEMENTS}

This work was supported by grants from the Délégation à la Recherche Clinique and Assistance Publique-Hôpitaux de Paris (AP-HP).

Data for this study were provided by the following members of the Groupe d'Etude des Lymphomes Digestifs: P. Le Coz (Villemomble), D. Dervichian (Longjumeau), C. Manole (St Germain en Laye), E. Abdini (Anglet), B. Zielinski, Z. Marjanovic, J. Bellanger, V. Haddad (Paris), G. Minier (Bussy Saint Georges), D. Kusielewicz (Le Blanc Mesnil), A. Delmer (Reims); Review Committee of Pathologists: J. Audouin, B. Fabiani. We wish to thank Maxime Leroy for his help with statistical analyses.

\section{AUTHOR CONTRIBUTIONS}

Concept and design: T.M., A.R. Data analysis and interpretation: T.M., A.R., P.J., N.C. Drafting of the manuscript: T.M., A.R., P.J., N.C. Critical revision of the manuscript for essential intellectual content: B.F., P.C. All authors read and approved the final manuscript.

\section{ORCID}

Tamara Matysiak-Budnik https://orcid.org/0000-0003-0780-6261

Philippe Jamet https://orcid.org/0000-0003-0592-9831

Nicolas Chapelle https://orcid.org/0000-0003-4834-9693

Bettina Fabiani https://orcid.org/0000-0003-1865-2587

Paul Coppo https://orcid.org/0000-0002-4618-2095

Agnès Ruskoné-Fourmestraux

https://orcid.org/0000-0001-5876-1716

\section{REFERENCES}

1. Matysiak-Budnik T, Jamet P, Fabiani B, et al. Primary intestinal B-cell lymphoma: a prospective multicentre clinical study of 91 cases. Dig Liver Dis 2013;45:947-952.

2. Fujishima F, Katsushima H, Fukuhara N, et al. Incidence rate, subtype frequency, and occurrence site of malignant lymphoma in the gastrointestinal tract: population-based analysis in Miyagi, Japan. Tohoku J Exp Med 2018;245:159165 .

3. Nakamura S, Matsumoto T, Takeshita M, et al. A clinicopathologic study of primary small intestine lymphoma: prognostic significance of mucosa-associated lymphoid tissue-derived lymphoma. Cancer 2000;88:286-294.

4. Yamamoto S, Nakase H, Yamashita K, et al. Gastrointestinal follicular lymphoma: review of the literature. J Gastroenterol 2010;45:370-388.

5. Na HY, Kim YA, Lee C, et al. Gastric follicular lymphoma: a report of 3 cases and a review of the literature. Oncol Lett 2018;16:741-748 
6. Takeuchi K, Iwamuro M, Imagawa A, et al. Primary follicular lymphoma of the duodenum with erosions as atypical macroscopic features. Case Rep Med 2012;2012:582607.

7. Fujimoto A, Akimoto T, Nakayama A, et al. A gastric follicular lymphoma presenting as a submucosal tumor with a diagnosis determined by endoscopic resection. Intern Med 2018;57:1849-1853.

8. Seki A, Iwamuro M, Yoshioka M, et al. Primary duodenal follicular lymphoma treated with rituximab monotherapy and followed-up for 15 years. Acta Med Okayama 2015;69:301-306

9. Amita K, Suma MN, Shankar SV, Premalata CS, Sanjay M. Primary follicular lymphoma of ileum [corrected] disguised as multiple miliary like lesions: a case report and review of literature. Indian J Pathol Microbiol 2018;61:446-449.

10. Sato Y, Ichimura K, Tanaka T, et al. Duodenal follicular lymphomas share common characteristics with mucosa-associated lymphoid tissue lymphomas. J Clin Pathol 2008;61:377381.

11. Sentani K, Maeshima AM, Nomoto J, et al. Follicular lymphoma of the duodenum: a clinicopathologic analysis of 26 cases. Jpn J Clin Oncol 2008;38:547-552.

12. Andraos T, Ayoub Z, Nastoupil L, et al. Early stage extranodal follicular lymphoma: characteristics, management, and outcomes. Clin Lymphoma Myeloma Leuk 2019;19:381-389.

13. Shia J, Teruya-Feldstein J, Pan D, et al. Primary follicular lymphoma of the gastrointestinal tract: a clinical and pathologic study of 26 cases. Am J Surg Pathol 2002;26:216-224.

14. Damaj G, Verkarre V, Delmer A, et al. Primary follicular lymphoma of the gastrointestinal tract: a study of 25 cases and a literature review. Ann Oncol 2003;14:623-629.

15. Schmatz AI, Streubel B, Kretschmer-Chott E, et al. Primary follicular lymphoma of the duodenum is a distinct mucosal/ submucosal variant of follicular lymphoma: a retrospective study of 63 cases. J Clin Oncol 2011;29:1445-1451.

16. Tari A, Asaoku H, Kunihiro M, Tanaka S, Fujihara M, Yoshino T. Clinical features of gastrointestinal follicular lymphoma: comparison with nodal follicular lymphoma and gastrointestinal MALT lymphoma. Digestion 2011;83:191197.

17. Takata K, Okada H, Ohmiya N, et al. Primary gastrointestinal follicular lymphoma involving the duodenal second portion is a distinct entity: a multicenter, retrospective analysis in Japan. Cancer Sci 2011;102:1532-1536.

18. Misdraji J, Harris NL, Hasserjian RP, Lauwers GY, Ferry JA. Primary follicular lymphoma of the gastrointestinal tract. Am J Surg Pathol 2011;35:1255-1263.

19. Takata K, Miyata-Takata T, Sato Y, et al. Gastrointestinal follicular lymphoma: current knowledge and future challenges. Pathol Int 2018;68:1-6.

20. Lewin KJ, Ranchod M, Dorfman RF. Lymphomas of the gastrointestinal tract: a study of 117 cases presenting with gastrointestinal disease. Cancer 1978;42:693-707.

21. Swerdlow SH, Campo E, Pileri SA, et al. The 2016 revision of the World Health Organization classification of lymphoid neoplasms. Blood 2016;127:2375-2390.

22. Musshoff K. Clinical staging classification of non-Hodgkin's lymphomas (author's transl). Strahlentherapie 1977;153:218221.

23. Freedman A, Jacobsen E. Follicular lymphoma: 2020 update on diagnosis and management. Am J Hematol 2020;95:316327.

24. Solal-Céligny P, Roy P, Colombat P, et al. Follicular lymphoma international prognostic index. Blood 2004;104:12581265.

25. Takata K, Miyata-Takata T, Sato Y, Yoshino T. Pathology of follicular lymphoma. J Clin Exp Hematop 2014;54:3-9.

26. Metser U, Hussey D, Murphy G. Impact of (18)F-FDG PET/ $\mathrm{CT}$ on the staging and management of follicular lymphoma. Br J Radiol 2014;87:20140360.

27. Dreyling M, Ghielmini M, Rule S, et al. Newly diagnosed and relapsed follicular lymphoma: ESMO Clinical Practice Guidelines for diagnosis, treatment and follow-up. Ann Oncol 2021;32:298-308.

28. Duffles Amarante G, Collins G, Rocha V. What do we know about duodenal-type follicular lymphoma? From pathological definition to treatment options. Br J Haematol 2020;188:831-837.

29. Marks E, Shi Y. Duodenal-type follicular lymphoma: a clinicopathologic review. Arch Pathol Lab Med 2018;142:542547.

30. Tari A, Kitadai Y, Mouri R, et al. Watch-and-wait policy versus rituximab-combined chemotherapy in Japanese patients with intestinal follicular lymphoma. J Gastroenterol Hepatol 2018;33:1461-1468.

31. Hauer-Jensen M, Denham JW, Andreyev HJ. Radiation enteropathy: pathogenesis, treatment and prevention. Nat Rev Gastroenterol Hepatol 2014;11:470-479.

32. Lightner AL, Shannon E, Gibbons MM, Russell MM. Primary gastrointestinal non-Hodgkin's lymphoma of the small and large intestines: a systematic review. J Gastrointest Surg 2016;20:827-839.

33. Madsen C, Plesner TL, Bentzen HH, et al. Real world data on histological transformation in patients with follicular lymphoma: incidence, clinico-pathological risk factors and outcome in a nationwide Danish cohort. Leuk Lymphoma 2020;61:2584-2594.

34. Foukas PG, Bisig B, de Leval L. Recent advances upper gastrointestinal lymphomas: molecular updates and diagnostic implications. Histopathology 2021;78:187-214.

35. Capelle LG, de Vries AC, Looman CW, et al. Gastric MALT lymphoma: epidemiology and high adenocarcinoma risk in 
a nation-wide study. Eur J Cancer 2008;44:2470-2476.

36. Palmela C, Fonseca C, Faria R, Baptista RB, Ribeiro S, Ferreira AO. Increased risk for metachronous gastric ad- enocarcinoma following gastric MALT lymphoma: a US population-based study. United European Gastroenterol J 2017;5:473-478. 